\title{
A Context-Aware Framework for an Intelligent Mall based on Recommender System
}

\author{
Yuxiang Ye ${ }^{\mathrm{a}}$, Run Zhao ${ }^{\mathrm{b}}$ and Dong Wang ${ }^{\mathrm{c}}$ \\ School of Software Engineering, Shanghai Jiao Tong University, Shanghai 200240, China \\ ayyxyutou@sjtu.edu.cn, b'zhaorun@cs.sjtu.edu.cn, cwangdong@cs.sjtu.edu.cn
}

Keywords: Context-aware, Intelligent Mall, Behavior Model, Recommend System.

\begin{abstract}
IntelliSense technology used in various fields are constantly expanding from the depth and breadth. Currently, most of the academic research on intelligent sensing systems are in specific fields such as health care, environmental monitoring, smart home. And less in-depth and practical research for smart shopping environment. In this paper, we design a recommendation system based on intelligent business district. First we design the architecture of the system which is efficient and scalable. Then raise the concept of behavior context model to describe all the situation in the intelligent mall.
\end{abstract}

\section{Introduction}

With the growing popularity of the Internet of Things [1], smart devices in a variety of applications in the field has been gradually playing an increasingly important role. The current context-aware system is mainly focused on medical, military, information retrieval, electronic commerce, smart home, mobile computing, environmental monitoring and other applications, and has made very satisfactory results. At the same time, the importance of contextual information has also been extensively studied and validated.

In the field of recommender systems, the majority of research focuses on the association between the user and the project [2], and less at this time to consider its geographical context (such as the user's location, behavior and time, etc.). However, in the mall scene intelligence, relying solely on the binary relationship between the user and the project, or the user, project, ternary relationships such user and cannot be effectively recommended.

This paper proposes a common scalable recommendation system framework using in intelligent mall. In the underlying of system works on data collation and data abstraction, shielding the heterogeneity of different devices. In the logical layer allows all types of recommender systems algorithm embedded or detached from the system in the form of hot-swap dynamically, the system has good scalability. In the service layer provides a unified data presenting interface. In the push layer implements the subscription and publication of information based on the user's needs.

\section{Related Work}

Context-aware recommendation system [3] by introducing contextual information recommendation system, in order to further improve the recommendation accuracy and user satisfaction, has important significance. Sensing technology is so advanced today, the mall has long been around all kinds of intelligent sensors [4]. Combine identity information and real-time behavior of customers, the amount of information has been a rich and complex enough, the amount of information as the data is sufficient to recommend a variety of methods, which offer customers a more personalized recommendation service. However, in the mall environment, customers obviously do not want to install a wide variety of applications on their mobile phones, so the establishment of a unified context-aware framework based on intelligent mall is necessary.

By analyzing the research status of context-aware systems [5], we can see that the context-aware system has a complex architecture of context information, as well as multiple module assembly is used for sensing collect context information, context information modeling, filtering, combining, 
using the inference engine of reasoning and displayed to the user. But overall, the system framework include perception, reasoning, push three parts.

\section{System Architecture}

We reference to the traditional hierarchical system architecture model, combined with the actual situation of smart shopping malls to optimize and improve the design of a framework for context-aware system based on smart shopping, as shown in Figure 1.

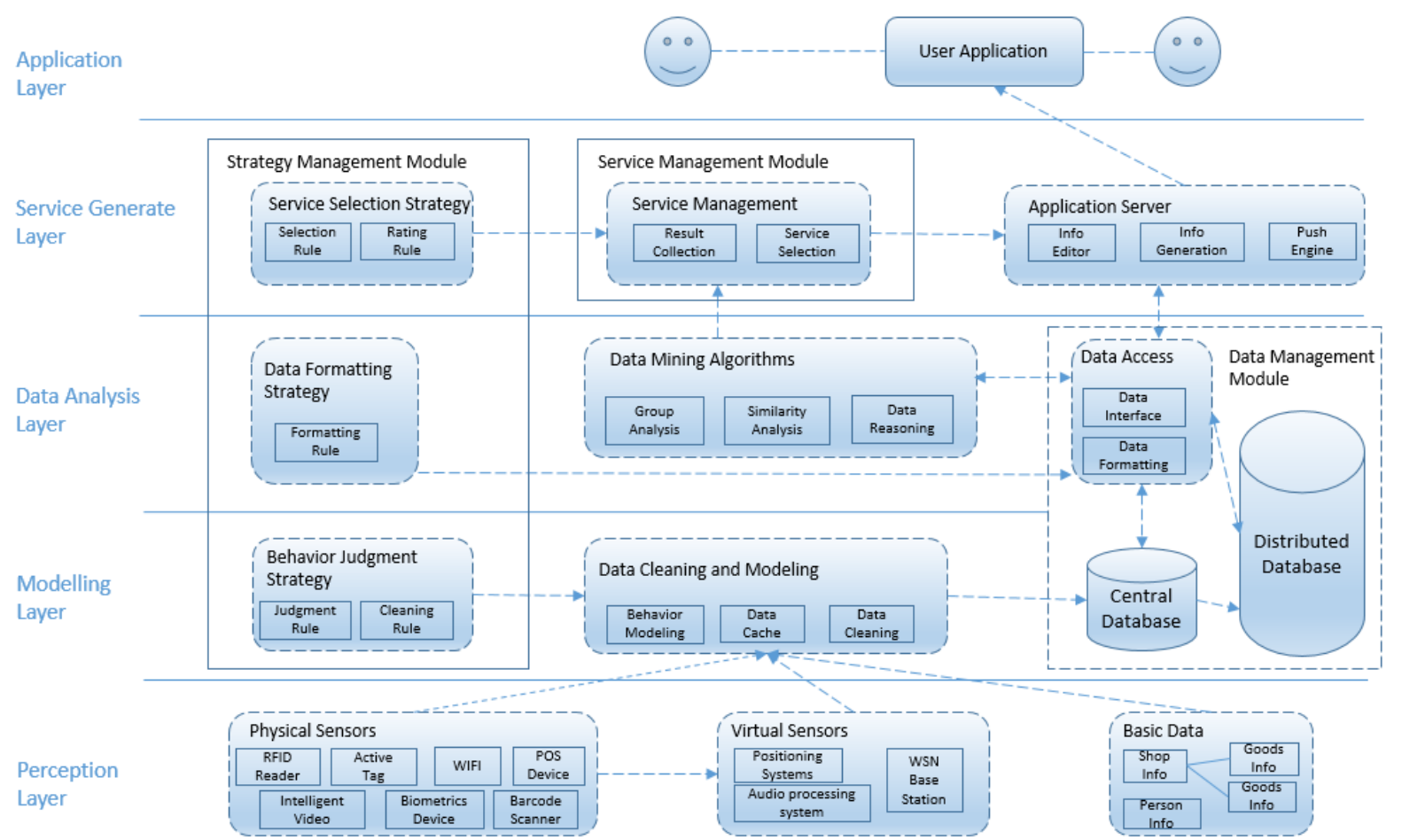

Fig. 1 the architecture of context-aware system based on intelligent mall

From the perspective of the logical hierarchy, the system comprises five layers: perception layer, model building layer, the layers of the data analysis, the service push layer and the application layer.

Perception layer: As the information source in intelligent mall, it access basic data and provide to system when needed.

Modeling layer: Data cleaning and integration from perception layer, so that the formation form the behavior path model.

Data analysis layer: Data access through a unified interface to obtain the behavior path information, and data mining analysis by specific algorithms.

Service generate layer: Consolidate information to the server application, push them to the system and manage the interaction with the server application.

Application layer: The customer use it. All the work other layers do are work for providing service to user.

From a management perspective, the system consists of three modules: data management module, strategy management module and service management module.

Data Management Module: A unified basic data storage and data access methods. Basic system data and user behavior data path integrated management. And can realize distributed data management system according to the amount of data changes.

Strategy Management Module: Protocol, strategies and algorithms which are system layers needed unified management and configuration, so that to enhance system scalability.

Service Management Module: Management system for various services, shielding the heterogeneity between the various services for the application layer. 


\section{Behavior Modeling}

This paper presents the concept of intelligent behavior mall environment context: a continuous period of time the user changes the change of location and behavior made in, constitute the context of the user's behavior. Behavior over time and determine the location of the user to make a determination within a mall, referred to herein as a behavioral point of the user. Collection of users patronize mall within a certain period of time to produce a series of actions points, referred to herein as the behavior of the path. By temporal information and behavioral information polymerization is a behavior point, a customer at the mall is one of the inevitable behavior patterns point activities, thus constituting a path in which a customer behavior in the mall.

Behavior Model Detail. Context-aware intelligent model-based shopping, mainly by three parts, namely, physical things, location and behavior. Things entities, including the people, goods and shops. That space-time location data, representing the position of an individual at a time where the mall. The last part is the behavior at ambient intelligence mall, we summarize the five main behaviors: stay, walking, shopping, browsing and sign in. Figure 2 shows the behavior context model.

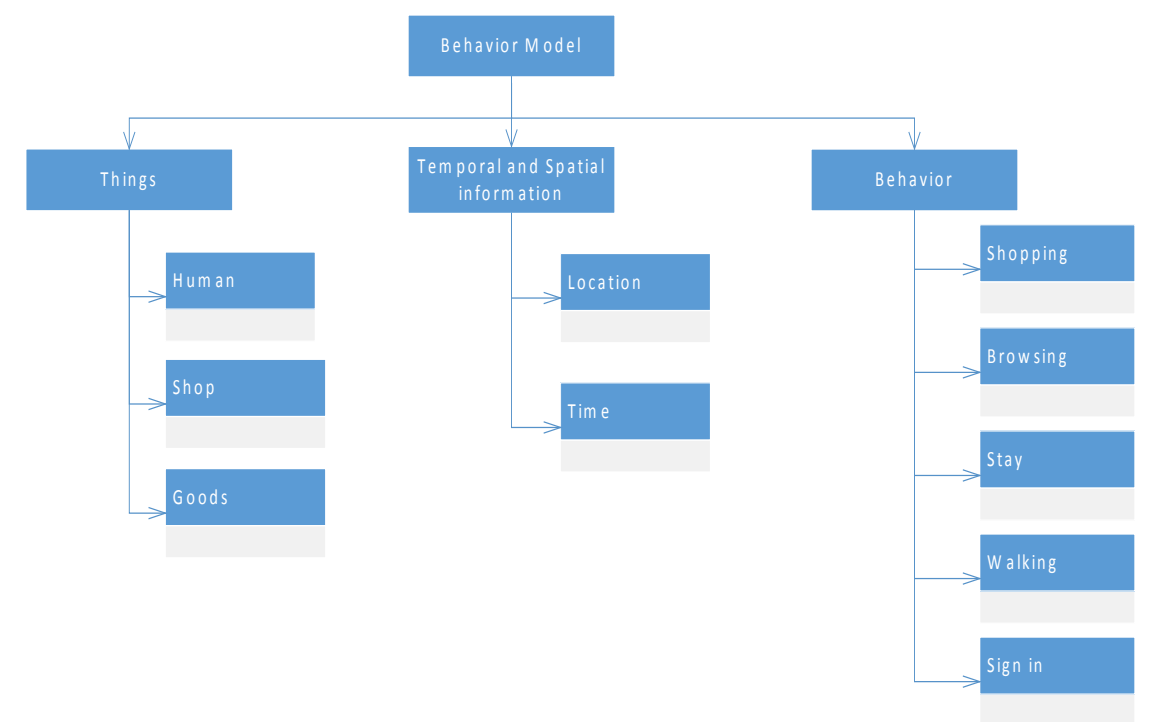

Fig. 2 Behavior context model

Behavior Judgment. Human behavior information is to rely on various types of sensors work together to get. Figure 3.x show how to determine a customer's behavior. Consumer behavior is as shopping malls POS machines, cash registers and user information combine to determine a customer's behavior is currently being consumption; stays behaviors is positioning system in obtaining the user's location, the user does not change the position of this information , then we can infer this point the user to stay in a certain position on the mall; Similarly, if a user is in a position to stay in the shop, and the user has no consumption in shops, then we can infer its ongoing behavior is viewed products; if the user's location is constantly changing over time, it indicates that the user is shopping in a mobile, which is a movement behaviors, and the amount of change in frequency of the user's location and the user according to the displacement of the acquisition, we can also speculate that the user is walking or on the run; when users actively using the application software, also possible attendance at some sites it deems more interested in, this is a behaviors of the user. Figure 3 shows how the system judge user behavior. 


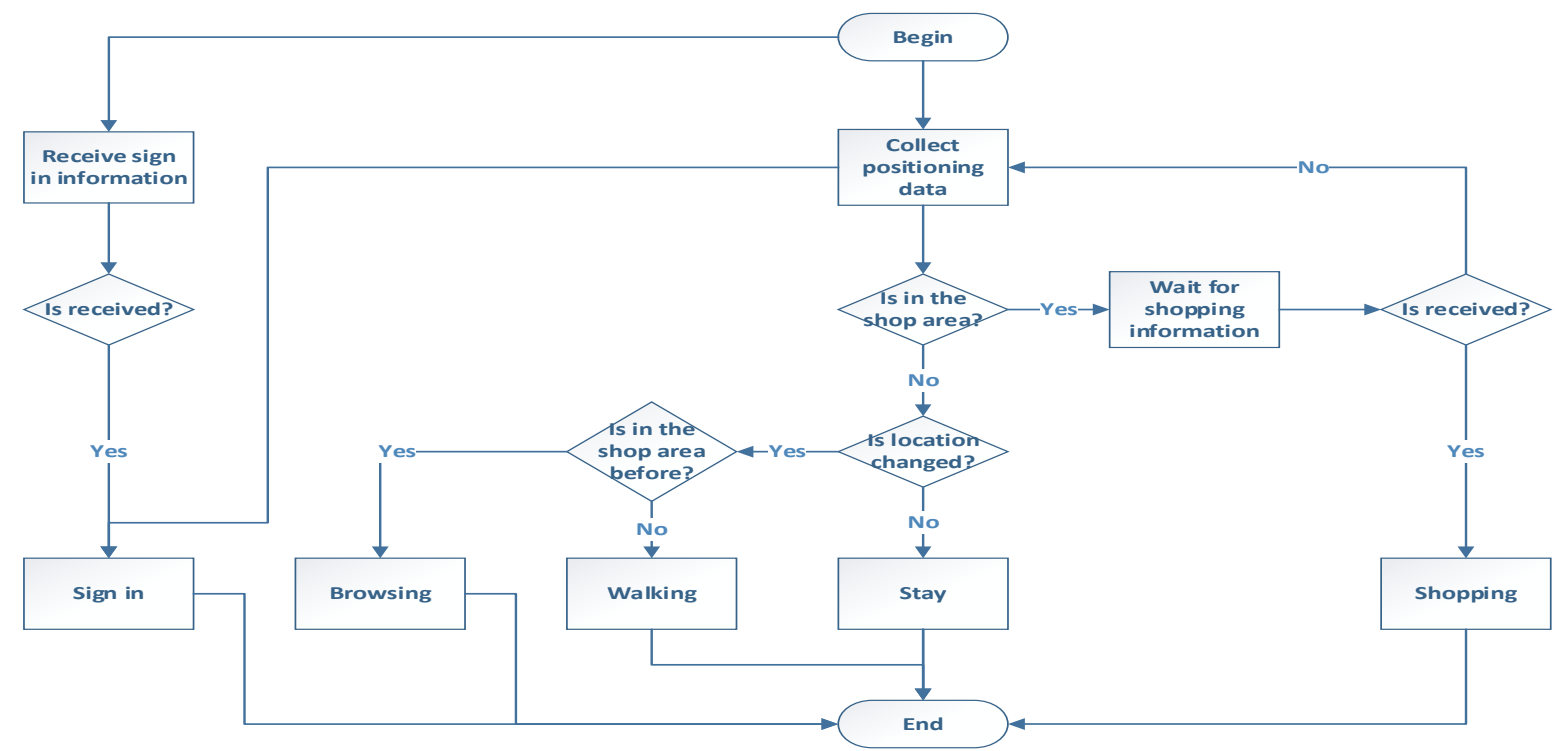

Fig. 3 Behavior judgment process

\section{Summary}

In this paper, the analysis of the environmental advantages of smart business district as well as challenges, put forward the corresponding design and build a framework based on context-aware intelligent district, and proposed a solution. Summary of the full text of the contribution of this study include the following two aspects:

First, sets up a context-aware framework based on intelligent shopping district, and gives a detailed design of all the layers and modules.

Second, proposes and designs the concept of user behavior context model and behavioral path, unifies the behavior of all users in the mall, and to give it time and space properties.

\section{References}

[1] Perera, C., et al. Context Aware Computing for the Internet of Things: A Survey. Communications Surveys \& Tutorials, IEEE 16(2014) p.414-454.

[2] Xu Jian Feng, Wang Dong. Object-oriented and ontology context-aware modeling based on XML, 2nd Int. Conference on Computer Science and Network Technology. (2012) p.1795-1800.

[3] Adomavicius G, Sankaranarayanan R, Sen S, Tuzhilin A. Incorporating contextual information in recommender systems using a multidimensional approach, ACM TOIS. (2005) p.103-145.

[4] Mehra, Pankaj. Context-Aware Computing: Beyond Search and Location-Based Services, IEEE Internet Computing (2012) p.12-16.

[5] Verbert, K., et al. Context-Aware Recommender Systems for Learning: A Survey and Future Challenges, Learning Technologies, IEEE Transactions on (2012) p.318-335. 